Acta Arith. 156(2012), no. 2, 123-141.

\title{
ON SUMS INVOLVING PRODUCTS OF THREE BINOMIAL COEFFICIENTS
}

\author{
ZHI-WeI SUN \\ Department of Mathematics, Nanjing University \\ Nanjing 210093, People's Republic of China \\ zwsun@nju.edu.cn \\ http://math.nju.edu.cn/ zwsun
}

Abstract. In this paper we mainly employ the Zeilberger algorithm to study congruences for sums of terms involving products of three binomial coefficients. Let $p>3$ be a prime. We prove that

$$
\sum_{k=0}^{p-1} \frac{\left(\begin{array}{c}
2 k \\
k
\end{array}\right)^{2}\left(\begin{array}{c}
2 k \\
k+d
\end{array}\right)}{64^{k}} \equiv 0 \quad\left(\bmod p^{2}\right)
$$

for all $d \in\{0, \ldots, p-1\}$ with $d \equiv(p+1) / 2(\bmod 2)$. If $p \equiv 1(\bmod 4)$ and $p=x^{2}+y^{2}$ with $x \equiv 1(\bmod 4)$ and $y \equiv 0(\bmod 2)$, then we show

$\sum_{k=0}^{p-1} \frac{\left(\begin{array}{c}2 k \\ k\end{array}\right)^{2}\left(\begin{array}{c}2 k \\ k+1\end{array}\right)}{(-8)^{k}} \equiv 2 p-2 x^{2}\left(\bmod p^{2}\right)$ and $\sum_{k=0}^{p-1} \frac{\left(\begin{array}{c}2 k \\ k\end{array}\right)\left(\begin{array}{c}2 k \\ k+1\end{array}\right)^{2}}{(-8)^{k}} \equiv-2 p\left(\bmod p^{2}\right)$

by means of determining $x \bmod p^{2}$ via

$$
(-1)^{(p-1) / 4} x \equiv \sum_{k=0}^{(p-1) / 2} \frac{k+1}{8^{k}}\left(\begin{array}{c}
2 k \\
k
\end{array}\right)^{2} \equiv \sum_{k=0}^{(p-1) / 2} \frac{2 k+1}{(-16)^{k}}\left(\begin{array}{c}
2 k \\
k
\end{array}\right)^{2} \quad\left(\bmod p^{2}\right) .
$$

We also solve the remaining open cases of Rodriguez-Villegas' conjectural congruences on

$$
\sum_{k=0}^{p-1} \frac{\left(\begin{array}{c}
2 k \\
k
\end{array}\right)^{2}\left(\begin{array}{c}
3 k \\
k
\end{array}\right)}{108^{k}}, \sum_{k=0}^{p-1} \frac{\left(\begin{array}{c}
2 k \\
k
\end{array}\right)^{2}\left(\begin{array}{c}
4 k \\
2 k
\end{array}\right)}{256^{k}}, \sum_{k=0}^{p-1} \frac{\left(\begin{array}{c}
2 k \\
k
\end{array}\right)\left(\begin{array}{c}
3 k \\
k
\end{array}\right)\left(\begin{array}{c}
6 k \\
3 k
\end{array}\right)}{12^{3 k}}
$$

modulo $p^{2}$.

2010 Mathematics Subject Classification. Primary 11B65; Secondary 05A10, 11A07, $11 \mathrm{E} 25$.

Keywords. Central binomial coefficients, super congruences, Zeilberger's algorithm, Schröder numbers, binary quadratic forms.

Supported by the National Natural Science Foundation (grant 11171140) of China and the PAPD of Jiangsu Higher Education Institutions. 


\section{INTRODUCTION}

Let $p$ be an odd prime. It is known that (see, e.g., S. Ahlgren [A], L. van Hamme $[\mathrm{vH}]$ and T. Ishikawa $[\mathrm{I}]$ )

$$
\begin{aligned}
& \sum_{k=0}^{(p-1) / 2}(-1)^{k}\left(\begin{array}{c}
-1 / 2 \\
k
\end{array}\right){ }^{3} \\
\equiv & \begin{cases}4 x^{2}-2 p\left(\bmod p^{2}\right) & \text { if } p=x^{2}+y^{2}(2 \nmid x \& 2 \mid y), \\
0\left(\bmod p^{2}\right) & \text { if } p \equiv 3(\bmod 4) .\end{cases}
\end{aligned}
$$

Clearly,

$$
\left(\begin{array}{c}
-1 / 2 \\
k
\end{array}\right)=\frac{\left(\begin{array}{c}
2 k \\
k
\end{array}\right)}{(-4)^{k}} \quad \text { for all } k \in \mathbb{N}=\{0,1,2, \ldots\}
$$

and

$$
\left(\begin{array}{c}
2 k \\
k
\end{array}\right)=\frac{(2 k) !}{(k !)^{2}} \equiv 0 \quad(\bmod p) \quad \text { for any } k=\frac{p+1}{2}, \ldots, p-1
$$

After his determination of $\sum_{k=0}^{p-1}\left(\begin{array}{c}2 k \\ k\end{array}\right) / m^{k} \bmod p^{2}$ (where $m \in \mathbb{Z}$ and $m \not \equiv$ $0(\bmod p))$ in $[\mathrm{Su} 1]$, the author $[\mathrm{Su} 2, \mathrm{Su} 3]$ posed some conjectures on $\sum_{k=0}^{p-1}\left(\begin{array}{c}2 k \\ k\end{array}\right)^{3} / m^{k} \bmod p^{2}$ with $m \in\{1,-8,16,-64,256,-512,4096\}$; for example, the author [Su2] conjectured that

$$
\sum_{k=0}^{p-1}\left(\begin{array}{c}
2 k \\
k
\end{array}\right)^{3} \equiv \begin{cases}4 x^{2}-2 p\left(\bmod p^{2}\right) & \text { if }\left(\frac{p}{7}\right)=1 \& p=x^{2}+7 y^{2}(x, y \in \mathbb{Z}) \\
0\left(\bmod p^{2}\right) & \text { if }\left(\frac{p}{7}\right)=-1, \text { i.e., } p \equiv 3,5,6(\bmod 7)\end{cases}
$$

where $(-)$ denotes the Legendre symbol. (It is known that if $\left(\frac{p}{7}\right)=1$ then $p=x^{2}+7 y^{2}$ for some $x, y \in \mathbb{Z}$; see, e.g., [C, p. 31].) Quite recently Z.-H. Sun [S2] made a certain progress on those conjectures; in particular, he proved (1.1) in the case $\left(\frac{p}{7}\right)=-1$ and confirmed the author's conjecture on $\sum_{k=0}^{p-1}\left(\begin{array}{c}2 k \\ k\end{array}\right)^{3} /(-8)^{k} \bmod p^{2}$.

Let $p=2 n+1$ be an odd prime. It is easy to see that for any $k=0, \ldots, n$ we have

$$
\left(\begin{array}{c}
n+k \\
2 k
\end{array}\right)=\frac{\prod_{j=1}^{k}\left(-(2 j-1)^{2}\right)}{4^{k}(2 k) !} \prod_{j=1}^{k}\left(1-\frac{p^{2}}{(2 j-1)^{2}}\right) \equiv \frac{\left(\begin{array}{c}
2 k \\
k
\end{array}\right)}{(-16)^{k}}\left(\bmod p^{2}\right)
$$

Based on this observation Z.-H. Sun [S2] studied the polynomial

$$
f_{n}(x)=\sum_{k=0}^{n}\left(\begin{array}{c}
n+k \\
2 k
\end{array}\right)\left(\begin{array}{c}
2 k \\
k
\end{array}\right)^{2} x^{k}
$$


and found the key identity

$$
f_{n}(x(x+1))=D_{n}(x)^{2}
$$

in his approach to (1.1), where

$$
D_{n}(x):=\sum_{k=0}^{n}\left(\begin{array}{c}
n+k \\
2 k
\end{array}\right)\left(\begin{array}{c}
2 k \\
k
\end{array}\right) x^{k}=\sum_{k=0}^{n}\left(\begin{array}{l}
n \\
k
\end{array}\right)\left(\begin{array}{c}
n+k \\
k
\end{array}\right) x^{k} .
$$

Note that the numbers $D_{n}=D_{n}(1)(n \in \mathbb{N})$ are the so-called central Delannoy numbers and $P_{n}(x):=D_{n}((x-1) / 2)$ is the Legendre polynomial of degree $n$.

Recall that the Catalan numbers are the integers defined by

$$
C_{n}=\frac{1}{n+1}\left(\begin{array}{c}
2 n \\
n
\end{array}\right)=\left(\begin{array}{c}
2 n \\
n
\end{array}\right)-\left(\begin{array}{c}
2 n \\
n+1
\end{array}\right) \quad(n \in \mathbb{N})
$$

while the Schröder numbers are given by

$$
S_{n}:=\sum_{k=0}^{n}\left(\begin{array}{c}
n+k \\
2 k
\end{array}\right) C_{k}=\sum_{k=0}^{n}\left(\begin{array}{l}
n \\
k
\end{array}\right)\left(\begin{array}{c}
n+k \\
k
\end{array}\right) \frac{1}{k+1} .
$$

We define the Schröder polynomial of degree $n$ by

$$
S_{n}(x):=\sum_{k=0}^{n}\left(\begin{array}{c}
n+k \\
2 k
\end{array}\right) C_{k} x^{k}
$$

For basic information about $D_{n}$ and $S_{n}$, the reader may consult [CHV], [Sl], [St, pp. 178 and 185], and [Su4].

In combinatorics, Zeilberger's algorithm developed in [Z] (see also Chapter 6 of [PWZ, pp.101-119]) is an algorithm which finds a polynomial recurrence for a terminating hypergeometric sum. For example, if we use Mathematica 7 and input $\mathrm{Zb}\left[\mathrm{Binomial}[\mathrm{n}, \mathrm{k}]^{\wedge} 3,\{\mathrm{k}, 0, \mathrm{n}\}, \mathrm{n}, 2\right]$, then we obtain the following second-order recurrence for $S(n)=\sum_{k=0}^{n}\left(\begin{array}{l}n \\ k\end{array}\right)^{3}$ :

$$
-8(n+1)^{2} S(n)-\left(7 n^{2}+21 n+16\right) S(n+1)+(n+2)^{2} S(n+2)=0 .
$$

Via the Schröder polynomials and the Zeilberger algorithm, we obtain the following result.

Theorem 1.1. Let $p$ be an odd prime.

(i) We have

$$
\sum_{k=0}^{p-1} \frac{\left(\begin{array}{c}
2 k \\
k
\end{array}\right)^{2}\left(\begin{array}{c}
2 k \\
k+d
\end{array}\right)}{64^{k}} \equiv 0 \quad\left(\bmod p^{2}\right)
$$


for all $d \in\{0,1, \ldots, p-1\}$ with $d \equiv(p+1) / 2(\bmod 2)$.

(ii) If $p \equiv 3(\bmod 4)$, then

$$
\sum_{k=0}^{p-1} \frac{\left(\begin{array}{c}
2 k \\
k
\end{array}\right)^{2}\left(\begin{array}{c}
2 k \\
k+1
\end{array}\right)}{64^{k}} \equiv\left(2 p+2-2^{p-1}\right)\left(\begin{array}{c}
(p-1) / 2 \\
(p+1) / 4
\end{array}\right)^{2} \quad\left(\bmod p^{2}\right)
$$

Now we state our second theorem the first part of which plays a key role in our proof of the second part.

Theorem 1.2. Let $p \equiv 1(\bmod 4)$ be a prime and write $p=x^{2}+y^{2}$ with $x \equiv 1(\bmod 4)$ and $y \equiv 0(\bmod 2)$.

(i) We can determine $x \bmod p^{2}$ in the following way:

$$
(-1)^{(p-1) / 4} x \equiv \sum_{k=0}^{(p-1) / 2} \frac{k+1}{8^{k}}\left(\begin{array}{c}
2 k \\
k
\end{array}\right)^{2} \equiv \sum_{k=0}^{(p-1) / 2} \frac{2 k+1}{(-16)^{k}}\left(\begin{array}{c}
2 k \\
k
\end{array}\right)^{2} \quad\left(\bmod p^{2}\right)
$$

Also,

$$
\begin{gathered}
\sum_{k=0}^{(p-1) / 2} \frac{\left(\begin{array}{c}
2 k \\
k
\end{array}\right) C_{k}}{8^{k}} \equiv-2 \sum_{k=0}^{p-1} \frac{k\left(\begin{array}{c}
2 k \\
k
\end{array}\right)^{2}}{8^{k}} \equiv(-1)^{(p-1) / 4}\left(2 x-\frac{p}{x}\right) \quad\left(\bmod p^{2}\right) \\
S_{(p-1) / 2} \equiv \sum_{k=0}^{(p-1) / 2} \frac{\left(\begin{array}{c}
2 k \\
k
\end{array}\right) C_{k}}{(-16)^{k}} \equiv-8 \sum_{k=0}^{(p-1) / 2} \frac{k\left(\begin{array}{c}
2 k \\
k
\end{array}\right)^{2}}{(-16)^{k}} \\
\equiv(-1)^{(p-1) / 4} 2\left(2 x-\frac{p}{x}\right) \quad\left(\bmod p^{2}\right) \\
\sum_{k=0}^{(p-1) / 2} \frac{k^{2}\left(\begin{array}{c}
2 k \\
k
\end{array}\right)^{2}}{8^{k}} \\
\sum_{(-1)^{(p-1) / 4}\left(x-\frac{3 p}{4 x}\right) \quad\left(\bmod p^{2}\right)}
\end{gathered}
$$

and

$$
\sum_{k=0}^{(p-1) / 2} \frac{k^{2}\left(\begin{array}{c}
2 k \\
k
\end{array}\right)^{2}}{(-16)^{k}} \equiv(-1)^{(p+3) / 4} \frac{p}{16 x} \quad\left(\bmod p^{2}\right) .
$$

(ii) We have

$$
\sum_{k=0}^{p-1} \frac{\left(\begin{array}{c}
2 k \\
k
\end{array}\right)^{2}\left(\begin{array}{c}
2 k \\
k+1
\end{array}\right)}{(-8)^{k}} \equiv 2 p-2 x^{2} \quad\left(\bmod p^{2}\right)
$$

and

$$
\sum_{k=0}^{p-1} \frac{\left(\begin{array}{c}
2 k \\
k
\end{array}\right)\left(\begin{array}{c}
2 k \\
k+1
\end{array}\right)^{2}}{(-8)^{k}} \equiv-2 p \quad\left(\bmod p^{2}\right)
$$


Remark 1.1. Let $p$ be an odd prime. We conjecture that

$$
\begin{aligned}
& \sum_{k=0}^{p-1} \frac{k+1}{8^{k}}\left(\begin{array}{c}
2 k \\
k
\end{array}\right)^{2}+\sum_{k=0}^{(p-1) / 2} \frac{2 k+1}{(-16)^{k}}\left(\begin{array}{c}
2 k \\
k
\end{array}\right)^{2} \\
\equiv & \begin{cases}2\left(\frac{2}{p}\right) x\left(\bmod p^{3}\right) & \text { if } p=x^{2}+y^{2}(4|x-1 \& 2| y), \\
0\left(\bmod p^{2}\right) & \text { if } p \equiv 3(\bmod 4) .\end{cases}
\end{aligned}
$$

Motivated by his study of Gaussian hypergeometric series and CalabiYau manifolds, in 2003 Rodriguez-Villegas [RV] raised some conjectures on congruences. In particular, he conjectured that for any prime $p>3$ we have

$$
\sum_{k=0}^{p-1} \frac{\left(\begin{array}{c}
2 k \\
k
\end{array}\right)^{2}\left(\begin{array}{c}
3 k \\
k
\end{array}\right)}{108^{k}} \equiv b(p) \quad\left(\bmod p^{2}\right), \quad \sum_{k=0}^{p-1} \frac{\left(\begin{array}{c}
2 k \\
k
\end{array}\right)^{2}\left(\begin{array}{c}
4 k \\
2 k
\end{array}\right)}{256^{k}} \equiv c(p) \quad\left(\bmod p^{2}\right),
$$

and

$$
\sum_{k=0}^{p-1} \frac{\left(\begin{array}{c}
2 k \\
k
\end{array}\right)\left(\begin{array}{c}
3 k \\
k
\end{array}\right)\left(\begin{array}{c}
6 k \\
3 k
\end{array}\right)}{12^{3 k}} \equiv\left(\frac{p}{3}\right) a(p) \quad\left(\bmod p^{2}\right)
$$

where

$$
\begin{gathered}
\sum_{n=1}^{\infty} a(n) q^{n}=q \prod_{n=1}^{\infty}\left(1-q^{4 n}\right)^{6}=\eta(4 z)^{6}, \\
\sum_{n=1}^{\infty} b(n) q^{n}=q \prod_{n=1}^{\infty}\left(1-q^{6 n}\right)^{3}\left(1-q^{2 n}\right)^{3}=\eta^{3}(6 z) \eta^{3}(2 z), \\
\sum_{n=1}^{\infty} c(n) q^{n}=q \prod_{n=1}^{\infty}\left(1-q^{n}\right)^{2}\left(1-q^{2 n}\right)\left(1-q^{4 n}\right)\left(1-q^{8 n}\right)^{2}=\eta^{2}(8 z) \eta(4 z) \eta(2 z) \eta^{2}(z),
\end{gathered}
$$

and the Dedekind $\eta$-function is given by

$$
\eta(z)=q^{1 / 24} \prod_{n=1}^{\infty}\left(1-q^{n}\right) \quad\left(\operatorname{Im}(z)>0 \text { and } q=e^{2 \pi i z}\right) .
$$

In 1892 F. Klein and R. Fricke [KF] proved that (see also [SB])

$$
a(p)= \begin{cases}4 x^{2}-2 p & \text { if } p \equiv 1(\bmod 4) \text { and } p=x^{2}+y^{2}(2 \nmid x), \\ 0 & \text { if } p \equiv 3(\bmod 4) .\end{cases}
$$

By $[\mathrm{SB}]$ we also have

$$
b(p)= \begin{cases}4 x^{2}-2 p & \text { if } p \equiv 1(\bmod 3) \text { and } p=x^{2}+3 y^{2} \text { with } x, y \in \mathbb{Z}, \\ 0 & \text { if } p \equiv 2(\bmod 3),\end{cases}
$$


and

$$
c(p)= \begin{cases}4 x^{2}-2 p & \text { if }\left(\frac{-2}{p}\right)=1 \text { and } p=x^{2}+2 y^{2} \text { with } x, y \in \mathbb{Z} \\ 0 & \text { if }\left(\frac{-2}{p}\right)=-1, \text { i.e., } p \equiv 5,7(\bmod 8)\end{cases}
$$

Via an advanced approach involving the $p$-adic Gamma function and Gauss and Jacobi sums (see K. Ono [O, Chapter 11] for an introduction to this method), E. Mortenson [M] managed to provide a partial solution of (1.14) and (1.15), with the following congruences still open:

$$
\begin{gathered}
\sum_{k=0}^{p-1} \frac{\left(\begin{array}{c}
2 k \\
k
\end{array}\right)^{2}\left(\begin{array}{c}
3 k \\
k
\end{array}\right)}{108^{k}} \equiv b(p)=0\left(\bmod p^{2}\right) \quad \text { if } p \equiv 5(\bmod 6), \\
\sum_{k=0}^{p-1} \frac{\left(\begin{array}{c}
2 k \\
k
\end{array}\right)^{2}\left(\begin{array}{c}
4 k \\
2 k
\end{array}\right)}{256^{k}} \equiv c(p)\left(\bmod p^{2}\right) \quad \text { if } p \equiv 3(\bmod 4), \\
\sum_{k=0}^{p-1} \frac{\left(\begin{array}{c}
2 k \\
k
\end{array}\right)\left(\begin{array}{c}
3 k \\
k
\end{array}\right)\left(\begin{array}{c}
6 k \\
3 k
\end{array}\right)}{12^{3 k}} \equiv-a(p)\left(\bmod p^{2}\right) \quad \text { if } p \equiv 5(\bmod 6) .
\end{gathered}
$$

Concerning (1.16)-(1.18), Mortenson's approach [M] only allowed him to show that for each of them the squares of both sides of the congruence are congruent modulo $p^{2}$.

Our following theorem confirms (1.16)-(1.18) and hence completes the proof of (1.14) and (1.15). So far, all conjectures of Rodriguez-Villegas [RV] involving at most three products of binomial coefficients have been proved!

Theorem 1.3. Let $p>3$ be a prime.

(i) Given $d \in\{0, \ldots, p-1\}$, we have

$$
\begin{aligned}
& \sum_{k=0}^{p-1} \frac{\left(\begin{array}{c}
2 k \\
k+d
\end{array}\right)\left(\begin{array}{c}
2 k \\
k
\end{array}\right)\left(\begin{array}{c}
3 k \\
k
\end{array}\right)}{108^{k}} \equiv 0\left(\bmod p^{2}\right) \quad \text { if } d \equiv \frac{1+\left(\frac{p}{3}\right)}{2}(\bmod 2), \\
& \sum_{k=0}^{p-1} \frac{\left(\begin{array}{c}
2 k \\
k+d
\end{array}\right)\left(\begin{array}{c}
2 k \\
k
\end{array}\right)\left(\begin{array}{c}
4 k \\
2 k
\end{array}\right)}{256^{k}} \equiv 0\left(\bmod p^{2}\right) \quad \text { if } d \equiv \frac{1+\left(\frac{-2}{p}\right)}{2}(\bmod 2), \\
& \sum_{k=0}^{p-1} \frac{\left(\begin{array}{c}
2 k \\
k+d
\end{array}\right)\left(\begin{array}{c}
3 k \\
k
\end{array}\right)\left(\begin{array}{c}
6 k \\
3 k
\end{array}\right)}{12^{3 k}} \equiv 0\left(\bmod p^{2}\right) \quad \text { if } d \equiv \frac{1+\left(\frac{-1}{p}\right)}{2}(\bmod 2) .
\end{aligned}
$$

(ii) If $p \equiv 3(\bmod 8)$ and $p=x^{2}+2 y^{2}$ with $x, y \in \mathbb{Z}$, then

$$
\sum_{k=0}^{p-1} \frac{\left(\begin{array}{c}
2 k \\
k
\end{array}\right)^{2}\left(\begin{array}{c}
4 k \\
2 k
\end{array}\right)}{256^{k}} \equiv 4 x^{2}-2 p \quad\left(\bmod p^{2}\right)
$$


(iii) If $p \equiv 5(\bmod 12)$ and $p=x^{2}+y^{2}$ with $2 \nmid x$ and $2 \mid y$, then

$$
\sum_{k=0}^{p-1} \frac{\left(\begin{array}{c}
2 k \\
k
\end{array}\right)\left(\begin{array}{c}
3 k \\
k
\end{array}\right)\left(\begin{array}{c}
6 k \\
3 k
\end{array}\right)}{12^{3 k}} \equiv 2 p-4 x^{2} \quad\left(\bmod p^{2}\right) \text {. }
$$

In the case $d=1$, Theorem 1.3(i) yields the following new result. (Note that $\left(\begin{array}{c}2 k \\ k\end{array}\right)\left(\begin{array}{c}3 k \\ k+1\end{array}\right)=2\left(\begin{array}{c}2 k \\ k+1\end{array}\right)\left(\begin{array}{c}3 k \\ k\end{array}\right)$ for any $k \in \mathbb{N}$.)

Corollary 1.1. Let $p>3$ be a prime. Then

$$
\begin{gathered}
\sum_{k=0}^{p-1} \frac{\left(\begin{array}{c}
2 k \\
k
\end{array}\right)^{2}\left(\begin{array}{c}
3 k \\
k+1
\end{array}\right)}{108^{k}} \equiv 0\left(\bmod p^{2}\right) \quad \text { if } p \equiv 1(\bmod 3), \\
\sum_{k=0}^{p-1} \frac{\left(\begin{array}{c}
4 k \\
2 k
\end{array}\right)\left(\begin{array}{c}
2 k \\
k
\end{array}\right)\left(\begin{array}{c}
2 k \\
k+1
\end{array}\right)}{256^{k}} \equiv 0\left(\bmod p^{2}\right) \quad \text { if } p \equiv 1,3(\bmod 8), \\
\sum_{k=0}^{p-1} \frac{\left(\begin{array}{c}
6 k \\
3 k
\end{array}\right)\left(\begin{array}{c}
3 k \\
k
\end{array}\right)\left(\begin{array}{c}
2 k \\
k+1
\end{array}\right)}{12^{3 k}} \equiv 0\left(\bmod p^{2}\right) \quad \text { if } p \equiv 1(\bmod 4) .
\end{gathered}
$$

We will prove Theorems 1.1-1.3 in Sections 2-4 respectively.

\section{Proof of Theorem 1.1}

Lemma 2.1. For any positive integer $n$ we have

$$
\sum_{k=1}^{n}\left(\begin{array}{c}
n+k \\
2 k
\end{array}\right)\left(\begin{array}{c}
2 k \\
k
\end{array}\right)\left(\begin{array}{c}
2 k \\
k+1
\end{array}\right) x^{k-1}(x+1)^{k+1}=n(n+1) S_{n}(x)^{2}
$$

Proof. Observe that

$$
S_{n}(x)^{2}=\sum_{k=0}^{n}\left(\begin{array}{c}
n+k \\
2 k
\end{array}\right) C_{k} x^{k} \sum_{l=0}^{n}\left(\begin{array}{c}
n+l \\
2 l
\end{array}\right) C_{l} x^{l}=\sum_{m=0}^{2 n} a_{m}(n) x^{m},
$$

where

$$
a_{m}(n):=\sum_{k=0}^{m}\left(\begin{array}{c}
n+k \\
2 k
\end{array}\right) C_{k}\left(\begin{array}{c}
n+m-k \\
2 m-2 k
\end{array}\right) C_{m-k} .
$$

Also, the coefficient of $x^{m}$ on the left-hand side of (2.1) coincides with

$$
\begin{aligned}
b_{m}(n) & :=\sum_{k=1}^{m+1}\left(\begin{array}{c}
n+k \\
2 k
\end{array}\right)\left(\begin{array}{c}
2 k \\
k
\end{array}\right)\left(\begin{array}{c}
2 k \\
k+1
\end{array}\right)\left(\begin{array}{c}
k+1 \\
m+1-k
\end{array}\right) \\
& =\sum_{k=0}^{m}\left(\begin{array}{c}
n+k+1 \\
2 k+2
\end{array}\right)\left(\begin{array}{c}
2 k+2 \\
k+1
\end{array}\right)\left(\begin{array}{c}
2 k+2 \\
k
\end{array}\right)\left(\begin{array}{c}
k+2 \\
m-k
\end{array}\right) .
\end{aligned}
$$


Thus, for the validity of (2.1) it suffices to show that $b_{m}(n)=n(n+1) a_{m}(n)$ for all $m=0,1, \ldots$ Obviously, $a_{0}(n)=1$ and $b_{0}(n)=n(n+1)$. Also, $a_{1}(n)=n(n+1)$ and $b_{1}(n)=n^{2}(n+1)^{2}$. By the Zeilberger algorithm via Mathematica 7 we find that both $u_{m}=a_{m}(n)$ and $u_{m}=b_{m}(n)$ satisfy the following recursion:

$$
\begin{aligned}
& (m+2)(m+3)(m+4) u_{m+2} \\
= & 2\left(2 m n^{2}+5 n^{2}+2 m n+5 n-m^{3}-6 m^{2}-11 m-6\right) u_{m+1} \\
& -(m+1)(m-2 n)(m+2 n+2) u_{m} .
\end{aligned}
$$

So $b_{m}(n)=n(n+1) a_{m}(n)$ for all $m \in \mathbb{N}$. This proves $(2.1)$.

Proof of Theorem 1.1. We first determine $\sum_{k=0}^{p-1}\left(\begin{array}{c}2 k \\ k\end{array}\right)^{2}\left(\begin{array}{c}2 k \\ k+1\end{array}\right) / 64^{k} \bmod p^{2}$ via Lemma 2.1, which actually led the author to the study of (1.5).

Recall the following combinatorial identity (cf. [Su2, (4.3)]):

$$
\sum_{k=0}^{n}\left(\begin{array}{c}
n+k \\
2 k
\end{array}\right) \frac{C_{k}}{(-2)^{k}}= \begin{cases}(-1)^{(n-1) / 2} C_{(n-1) / 2} / 2^{n} & \text { if } 2 \nmid n, \\
0 & \text { if } 2 \mid n .\end{cases}
$$

Set $n=(p-1) / 2$. Applying (2.1) with $x=-1 / 2$ we get

$$
\sum_{k=1}^{n}\left(\begin{array}{c}
n+k \\
2 k
\end{array}\right)\left(\begin{array}{c}
2 k \\
k
\end{array}\right)\left(\begin{array}{c}
2 k \\
k+1
\end{array}\right) \frac{1}{(-2)^{k-1} 2^{k+1}}=n(n+1) S_{n}\left(-\frac{1}{2}\right)^{2} .
$$

Thus, with the helps from (1.2) and (2.2), we have

$$
\begin{aligned}
\sum_{k=0}^{p-1} \frac{\left(\begin{array}{c}
2 k \\
k
\end{array}\right)^{2}\left(\begin{array}{c}
2 k \\
k+1
\end{array}\right)}{64^{k}} & \equiv \sum_{k=1}^{n}\left(\begin{array}{c}
n+k \\
2 k
\end{array}\right)\left(\begin{array}{c}
2 k \\
k
\end{array}\right)\left(\begin{array}{c}
2 k \\
k+1
\end{array}\right) \frac{1}{(-4)^{k}} \\
& =-n(n+1) S_{n}\left(-\frac{1}{2}\right)^{2} \\
& \equiv \begin{cases}0\left(\bmod p^{2}\right) & \text { if } p \equiv 1(\bmod 4) \\
C_{(n-1) / 2}^{2} / 2^{2 n+2}\left(\bmod p^{2}\right) & \text { if } p \equiv 3(\bmod 4) .\end{cases}
\end{aligned}
$$

Therefore $(1.5)$ with $d=1$ holds if $p \equiv 1(\bmod 4)$. In the case $p \equiv 3$ $(\bmod 4)$, clearly

$$
\begin{aligned}
\frac{C_{(n-1) / 2}^{2}}{2^{2 n+2}} & =\frac{\left(\left(\begin{array}{c}
(p-1) / 2 \\
(p+1) / 4
\end{array}\right) \frac{2}{p-1}\right)^{2}}{4 \times 2^{p-1}} \\
& \equiv \frac{1}{(1-2 p)\left(1+p q_{p}(2)\right)}\left(\begin{array}{c}
(p-1) / 2 \\
(p+1) / 4
\end{array}\right)^{2} \\
& \equiv\left(1+2 p-p q_{p}(2)\right)\left(\begin{array}{c}
(p-1) / 2 \\
(p+1) / 4
\end{array}\right)^{2}\left(\bmod p^{2}\right)
\end{aligned}
$$


where $q_{p}(2)=\left(2^{p-1}-1\right) / p$, and hence (1.6) holds.

For $d=0,1,2, \ldots$ set

$$
u_{d}=\sum_{k=0}^{p-1} \frac{\left(\begin{array}{c}
2 k \\
k
\end{array}\right)^{2}\left(\begin{array}{c}
2 k \\
k+d
\end{array}\right)}{64^{k}}=\sum_{d \leqslant k<p} \frac{\left(\begin{array}{c}
2 k \\
k
\end{array}\right)^{2}\left(\begin{array}{c}
2 k \\
k+d
\end{array}\right)}{64^{k}}
$$

By the Zeilberger algorithm we find the recursion

$$
(2 d+1)^{2} u_{d}-(2 d+3)^{2} u_{d+2}=\frac{(2 p-1)^{2}(d+1)}{64^{p-1} p}\left(\begin{array}{c}
2 p \\
p+d+1
\end{array}\right)\left(\begin{array}{c}
2 p-2 \\
p-1
\end{array}\right)^{2} .
$$

Note that

$$
\left(\begin{array}{c}
2 p-2 \\
p-1
\end{array}\right)=p C_{p-1} \equiv 0 \quad(\bmod p)
$$

If $0 \leqslant d<p-2$, then

$$
\left(\begin{array}{c}
2 p \\
p+d+1
\end{array}\right)=\frac{2 p}{p+d+1}\left(\begin{array}{c}
2 p-1 \\
p+d
\end{array}\right) \equiv 0 \quad(\bmod p)
$$

and hence

$$
(2 d+1)^{2} u_{d} \equiv(2 d+3)^{2} u_{d+2} \quad\left(\bmod p^{2}\right) .
$$

For $d \in\{0, \ldots, p-3\}$ with $d \equiv(p+1) / 2(\bmod 2)$, clearly $p \neq 2 d+1<2 p$ and hence

$$
u_{d+2} \equiv 0 \quad\left(\bmod p^{2}\right) \Longrightarrow u_{d} \equiv 0 \quad\left(\bmod p^{2}\right) .
$$

If $d \in\{p-1, p-2\}$ and $d \equiv(p+1) / 2(\bmod 2)$, then $d \geqslant(p+1) / 2$ and hence $u_{d} \equiv 0\left(\bmod p^{2}\right)$. So $(1.5)$ holds for all $d \in\{0, \ldots, p-1\}$ with $d \equiv(p+1) / 2(\bmod 2)$.

So far we have completed the proof of Theorem 1.1.

\section{Proof of Theorem 1.2}

Lemma 3.1. For any $n \in \mathbb{N}$ we have

$$
\sum_{k=0}^{n}\left(\begin{array}{c}
2 k \\
k
\end{array}\right)^{3}\left(\begin{array}{c}
k \\
n-k
\end{array}\right)(-16)^{n-k}=\sum_{k=0}^{n}\left(\begin{array}{c}
2 k \\
k
\end{array}\right)^{2}\left(\begin{array}{c}
2(n-k) \\
n-k
\end{array}\right)^{2}
$$

Proof. For $n=0,1$, both sides of (3.1) take the values 1 and 8 respectively. Let $u_{n}$ denote the left-hand side of (3.1) or the right-hand side of (3.1). Applying the Zeilberger algorithm via Mathematica 7, we obtain the recursion

$$
(n+2)^{3} u_{n+2}=8(2 n+3)\left(2 n^{2}+6 n+5\right) u_{n+1}-256(n+1)^{3} u_{n}(n \in \mathbb{N}) .
$$

So, by induction (3.1) holds for all $n=0,1,2, \ldots$. 
Lemma 3.2. Let $p$ be an odd prime. Then

$$
\begin{aligned}
& \sum_{n=0}^{p-1} \frac{n+1}{8^{n}} \sum_{k=0}^{n}\left(\begin{array}{c}
2 k \\
k
\end{array}\right)^{2}\left(\begin{array}{c}
2(n-k) \\
n-k
\end{array}\right)^{2} \\
\equiv & \sum_{n=0}^{p-1} \frac{2 n+1}{(-16)^{n}} \sum_{k=0}^{n}\left(\begin{array}{c}
2 k \\
k
\end{array}\right)^{2}\left(\begin{array}{c}
2(n-k) \\
n-k
\end{array}\right)^{2} \\
\equiv & p\left(\frac{-1}{p}\right)\left(\bmod p^{3}\right) .
\end{aligned}
$$

Proof. In view of Lemma 3.1, we have

$$
\begin{aligned}
& \sum_{n=0}^{p-1} \frac{n+1}{8^{n}} \sum_{k=0}^{n}\left(\begin{array}{c}
2 k \\
k
\end{array}\right)^{2}\left(\begin{array}{c}
2(n-k) \\
n-k
\end{array}\right)^{2} \\
= & \sum_{n=0}^{p-1} \frac{n+1}{8^{n}} \sum_{k=0}^{n}\left(\begin{array}{c}
2 k \\
k
\end{array}\right)^{3}\left(\begin{array}{c}
k \\
n-k
\end{array}\right)(-16)^{n-k} \\
= & \sum_{k=0}^{p-1} \frac{\left(\begin{array}{c}
2 k \\
k
\end{array}\right)^{3}}{8^{k}} \sum_{j=0}^{p-1-k}(k+j+1)\left(\begin{array}{c}
k \\
j
\end{array}\right) \frac{(-16)^{j}}{8^{j}} \\
\equiv & \sum_{k=0}^{(p-1) / 2} \frac{\left(\begin{array}{c}
2 k \\
k
\end{array}\right)^{3}}{8^{k}}\left((k+1) \sum_{j=0}^{k}\left(\begin{array}{c}
k \\
j
\end{array}\right)(-2)^{j}-2 k \sum_{j=1}^{k}\left(\begin{array}{c}
k-1 \\
j-1
\end{array}\right)(-2)^{j-1}\right) \\
= & \sum_{k=0}^{(p-1) / 2} \frac{\left(\begin{array}{c}
2 k \\
k
\end{array}\right)^{3}}{8^{k}}\left((k+1)(-1)^{k}-2 k(-1)^{k-1}\right) \\
\equiv & \sum_{k=0}^{p-1} \frac{3 k+1}{(-8)^{k}}\left(\begin{array}{c}
2 k \\
k
\end{array}\right)^{3}\left(\bmod p^{3}\right) .
\end{aligned}
$$

In [Su3] the author conjectured that

$$
\sum_{k=0}^{p-1} \frac{3 k+1}{(-8)^{k}}\left(\begin{array}{c}
2 k \\
k
\end{array}\right)^{3} \equiv p\left(\frac{-1}{p}\right)+p^{3} E_{p-3} \quad\left(\bmod p^{4}\right)
$$

provided $p>3$, where $E_{0}, E_{1}, E_{2}, \ldots$ are Euler numbers given by

$$
E_{0}=1 \text { and } \sum_{\substack{k=0 \\
2 \mid k}}^{n}\left(\begin{array}{l}
n \\
k
\end{array}\right) E_{n-k}=0 \quad(n=1,2,3, \ldots) \text {. }
$$

The last congruence is still open but [GZ] confirmed that

$$
\sum_{k=0}^{p-1} \frac{3 k+1}{(-8)^{k}}\left(\begin{array}{c}
2 k \\
k
\end{array}\right)^{3} \equiv p\left(\frac{-1}{p}\right) \quad\left(\bmod p^{3}\right) .
$$


So we have

$$
\sum_{n=0}^{p-1} \frac{n+1}{8^{n}} \sum_{k=0}^{n}\left(\begin{array}{c}
2 k \\
k
\end{array}\right)^{2}\left(\begin{array}{c}
2(n-k) \\
n-k
\end{array}\right)^{2} \equiv p\left(\frac{-1}{p}\right) \quad\left(\bmod p^{3}\right)
$$

Similarly,

$$
\begin{aligned}
& \sum_{n=0}^{p-1} \frac{2 n+1}{(-16)^{n}} \sum_{k=0}^{n}\left(\begin{array}{c}
2 k \\
k
\end{array}\right)^{2}\left(\begin{array}{c}
2(n-k) \\
n-k
\end{array}\right)^{2} \\
= & \sum_{n=0}^{p-1} \frac{2 n+1}{(-16)^{n}} \sum_{k=0}^{n}\left(\begin{array}{c}
2 k \\
k
\end{array}\right)^{3}\left(\begin{array}{c}
k \\
n-k
\end{array}\right)(-16)^{n-k} \\
\equiv & \sum_{k=0}^{(p-1) / 2} \frac{\left(\begin{array}{c}
2 k \\
k
\end{array}\right)^{3}}{(-16)^{k}}\left((2 k+1) \sum_{j=0}^{k}\left(\begin{array}{c}
k \\
j
\end{array}\right)+2 k \sum_{j=1}^{k}\left(\begin{array}{c}
k-1 \\
j-1
\end{array}\right)\right) \\
\equiv & \sum_{k=0}^{p-1} \frac{3 k+1}{(-8)^{k}}\left(\begin{array}{c}
2 k \\
k
\end{array}\right)^{3} \equiv p\left(\frac{-1}{p}\right)\left(\bmod p^{3}\right) .
\end{aligned}
$$

This concludes the proof.

Lemma 3.3. Let $p$ be an odd prime. Then

$$
\begin{aligned}
2 \sum_{k=0}^{(p-1) / 2} \frac{k\left(\begin{array}{c}
2 k \\
k
\end{array}\right)^{2}}{8^{k}}+\sum_{k=0}^{(p-1) / 2} \frac{\left(\begin{array}{c}
2 k \\
k
\end{array}\right) C_{k}}{8^{k}} & \equiv 2 p^{2}\left(\frac{2}{p}\right)\left(\bmod p^{3}\right), \\
8 \sum_{k=0}^{(p-1) / 2} \frac{k\left(\begin{array}{c}
2 k \\
k
\end{array}\right)^{2}}{(-16)^{k}}+\sum_{k=0}^{(p-1) / 2} \frac{\left(\begin{array}{c}
2 k \\
k
\end{array}\right) C_{k}}{(-16)^{k}} & \equiv 2 p^{2}\left(\frac{-1}{p}\right)\left(\bmod p^{3}\right), \\
\sum_{k=0}^{(p-1) / 2}\left(2 k^{2}+4 k+1\right) \frac{\left(\begin{array}{c}
2 k \\
k
\end{array}\right)^{2}}{8^{k}} & \equiv p^{2}\left(\frac{2}{p}\right)\left(\bmod p^{3}\right), \\
\sum_{k=0}^{(p-1) / 2}\left(8 k^{2}+4 k+1\right) \frac{\left(\begin{array}{c}
2 k \\
k
\end{array}\right)^{2}}{(-16)^{k}} & \equiv p^{2}\left(\frac{-1}{p}\right)\left(\bmod p^{3}\right) .
\end{aligned}
$$


Proof. By induction, for every $n=0,1,2, \ldots$ we have

$$
\begin{aligned}
\sum_{k=0}^{n}\left(2 k+\frac{1}{k+1}\right) \frac{\left(\begin{array}{c}
2 k \\
k
\end{array}\right)^{2}}{8^{k}} & =\frac{(2 n+1)^{2}}{(n+1) 8^{n}}\left(\begin{array}{c}
2 n \\
n
\end{array}\right)^{2}, \\
\sum_{k=0}^{n}\left(8 k+\frac{1}{k+1}\right) \frac{\left(\begin{array}{c}
2 k \\
k
\end{array}\right)^{2}}{(-16)^{k}} & =\frac{(2 n+1)^{2}}{(n+1)(-16)^{n}}\left(\begin{array}{c}
2 n \\
n
\end{array}\right)^{2}, \\
\sum_{k=0}^{n}\left(2 k^{2}+4 k+1\right) \frac{\left(\begin{array}{c}
2 k \\
k
\end{array}\right)^{2}}{8^{k}} & =\frac{(2 n+1)^{2}}{8^{n}}\left(\begin{array}{c}
2 n \\
n
\end{array}\right)^{2}, \\
\sum_{k=0}^{n}\left(8 k^{2}+4 k+1\right) \frac{\left(\begin{array}{c}
2 k \\
k
\end{array}\right)^{2}}{(-16)^{k}} & =\frac{(2 n+1)^{2}}{(-16)^{n}}\left(\begin{array}{c}
2 n \\
n
\end{array}\right)^{2} .
\end{aligned}
$$

Applying these identities with $n=(p-1) / 2$ we immediately get the desired congruences.

Let $p \equiv 1(\bmod 4)$ be a prime and write $p=x^{2}+y^{2}$ with $x \equiv 1(\bmod 4)$ and $y \equiv 0(\bmod 2)$. In 1828 Gauss showed the congruence $\left(\begin{array}{c}(p-1) / 2 \\ (p-1) / 4\end{array}\right) \equiv 2 x$ $(\bmod p)$. In 1986, S. Chowla, B. Dwork and R. J. Evans [CDE] used Gauss and Jacobi sums to prove that

$$
\left(\begin{array}{l}
(p-1) / 2 \\
(p-1) / 4
\end{array}\right) \equiv \frac{2^{p-1}+1}{2}\left(2 x-\frac{p}{2 x}\right) \quad\left(\bmod p^{2}\right)
$$

which was first conjectured by F. Beukers. (See also [BEW, Chapter 9] and $[\mathrm{HW}$ ] for further related results.) In 2009, the author (see [Su2]) conjectured that

$$
\sum_{k=0}^{(p-1) / 2} \frac{\left(\begin{array}{c}
2 k \\
k
\end{array}\right)^{2}}{8^{k}} \equiv \sum_{k=0}^{(p-1) / 2} \frac{\left(\begin{array}{c}
2 k \\
k
\end{array}\right)^{2}}{(-16)^{k}} \equiv(-1)^{(p-1) / 4}\left(2 x-\frac{p}{2 x}\right) \quad\left(\bmod p^{2}\right)
$$

and this was confirmed by Z.-H. Sun [S1] via (3.2) and the Legendre polynomials.

Proof of Theorem 1.2(i). By (1.2),

$$
S_{(p-1) / 2} \equiv \sum_{k=0}^{(p-1) / 2} \frac{\left(\begin{array}{c}
2 k \\
k
\end{array}\right) C_{k}}{(-16)^{k}} \quad\left(\bmod p^{2}\right)
$$

In view of this and Lemma 3.3 and (3.3), it suffices to show (1.7). 
As $p \mid\left(\begin{array}{c}2 k \\ k\end{array}\right)$ for all $k=(p+1) / 2, \ldots, p-1$, we have

$$
\begin{aligned}
& \sum_{n=0}^{p-1} \frac{n+1}{8^{n}} \sum_{k=0}^{n}\left(\begin{array}{c}
2 k \\
k
\end{array}\right)^{2}\left(\begin{array}{c}
2(n-k) \\
n-k
\end{array}\right)^{2} \\
= & \sum_{k=0}^{p-1} \frac{\left(\begin{array}{c}
2 k \\
k
\end{array}\right)^{2}}{8^{k}} \sum_{n=k}^{p-1} \frac{n+1}{8^{n-k}}\left(\begin{array}{c}
2(n-k) \\
n-k
\end{array}\right)^{2} \\
= & \sum_{k=0}^{p-1} \frac{\left(\begin{array}{c}
2 k \\
k
\end{array}\right)^{2}}{8^{k}} \sum_{j=0}^{p-1-k} \frac{k+j+1}{8^{j}}\left(\begin{array}{c}
2 j \\
j
\end{array}\right)^{2} \\
\equiv & \sum_{k=0}^{(p-1) / 2} \frac{\left(\begin{array}{c}
2 k \\
k
\end{array}\right)^{2}}{8^{k}} \sum_{j=0}^{(p-1) / 2} \frac{(k+1)+(j+1)-1}{8^{j}}\left(\begin{array}{c}
2 j \\
j
\end{array}\right)^{2} \\
= & 2 \sum_{k=0}^{(p-1) / 2} \frac{\left(\begin{array}{c}
2 k \\
k
\end{array}\right)}{8^{k}} \sum_{j=0}^{(p-1) / 2} \frac{(j+1)\left(\begin{array}{c}
2 j \\
j
\end{array}\right)^{2}}{8^{j}}-\left(\sum_{k=0}^{p-1} \frac{\left(\begin{array}{c}
2 k \\
k
\end{array}\right)^{2}}{8^{k}}\right)^{2}\left(\bmod p^{2}\right) .
\end{aligned}
$$

Similarly,

$$
\begin{aligned}
& \sum_{n=0}^{p-1} \frac{2 n+1}{(-16)^{n}} \sum_{k=0}^{n}\left(\begin{array}{c}
2 k \\
k
\end{array}\right)^{2}\left(\begin{array}{c}
2(n-k) \\
n-k
\end{array}\right)^{2} \\
\equiv & 2 \sum_{k=0}^{(p-1) / 2} \frac{\left(\begin{array}{c}
2 k \\
k
\end{array}\right)^{2}}{(-16)^{k}} \sum_{j=0}^{(p-1) / 2} \frac{(2 j+1)\left(\begin{array}{c}
2 j \\
j
\end{array}\right)^{2}}{(-16)^{j}}-\left(\sum_{k=0}^{p-1} \frac{\left(\begin{array}{c}
2 k \\
k
\end{array}\right)^{2}}{(-16)^{k}}\right)^{2}\left(\bmod p^{2}\right) .
\end{aligned}
$$

Combining these with Lemma 3.2 and (3.3), we immediately obtain (1.7).

Lemma 3.4. Let $p \equiv 1(\bmod 4)$ be a prime. Write $p=x^{2}+y^{2}$ with $x \equiv 1(\bmod 4)$ and $y \equiv 0(\bmod 2)$. Then

$$
D_{(p-1) / 2} \equiv(-1)^{(p-1) / 4}\left(2 x-\frac{p}{2 x}\right) \quad\left(\bmod p^{2}\right) .
$$

Proof. By (1.2),

$$
D_{(p-1) / 2} \equiv \sum_{k=0}^{(p-1) / 2} \frac{\left(\begin{array}{c}
2 k \\
k
\end{array}\right)^{2}}{(-16)^{k}} \quad\left(\bmod p^{2}\right) .
$$

So (3.4) follows from (3.3).

Remark 3.1. If $p$ is a prime with $p \equiv 3(\bmod 4)$, then $n=(p-1) / 2$ is odd and hence

$$
\begin{aligned}
D_{n} & \equiv \sum_{k=0}^{n}(-1)^{k} \frac{\left(\begin{array}{c}
2 k \\
k
\end{array}\right)^{2}}{16^{k}}=\sum_{k=0}^{n}(-1)^{k}\left(\begin{array}{c}
-1 / 2 \\
k
\end{array}\right)^{2} \\
& \equiv \sum_{k=0}^{n}(-1)^{k}\left(\begin{array}{l}
n \\
k
\end{array}\right)^{2}=\sum_{k=0}^{n}(-1)^{n-k}\left(\begin{array}{l}
n \\
k
\end{array}\right)^{2}=0(\bmod p) .
\end{aligned}
$$


The following result was conjectured by the author [Su2] and confirmed by Z.-H. Sun [S2].

Lemma 3.5. Let $p$ be an odd prime. Then

$$
\sum_{k=0}^{p-1} \frac{\left(\begin{array}{c}
2 k \\
k
\end{array}\right)^{3}}{(-8)^{k}} \equiv \begin{cases}4 x^{2}-2 p\left(\bmod p^{2}\right) & \text { if } 4 \mid p-1 \& p=x^{2}+y^{2}(2 \nmid x), \\
0\left(\bmod p^{2}\right) & \text { if } p \equiv 3(\bmod 4) .\end{cases}
$$

Remark 3.2. Fix an odd prime $p=2 n+1$. By (1.2) and (1.3) we have

$$
\sum_{k=0}^{p-1} \frac{\left(\begin{array}{c}
2 k \\
k
\end{array}\right)^{3}}{(-8)^{k}} \equiv \sum_{k=0}^{n}\left(\begin{array}{c}
n+k \\
2 k
\end{array}\right)\left(\begin{array}{c}
2 k \\
k
\end{array}\right)^{2} 2^{k}=D_{n}^{2} \quad\left(\bmod p^{2}\right) .
$$

Hence (3.5) follows from Lemma 3.4 and Remark 3.1.

Lemma 3.6. For any positive integer $n$ we have

$$
\sum_{k=0}^{n}\left(\begin{array}{c}
n+k \\
2 k
\end{array}\right)\left(\begin{array}{c}
2 k \\
k
\end{array}\right)^{2} \frac{2 k+1}{(k+1)^{2}} x^{k}(x+1)^{k+1}=\frac{S_{n}(x)}{2}\left(D_{n-1}(x)+D_{n+1}(x)\right) .
$$

Proof. Note that

$$
S_{n}(x)\left(D_{n-1}(x)+D_{n+1}(x)\right)=\sum_{m=0}^{2 n+1} c_{m}(n) x^{m}
$$

where

$$
\begin{aligned}
& c_{m}(n)=\sum_{k=0}^{m}\left(\begin{array}{c}
n+k \\
2 k
\end{array}\right) C_{k}\left(\begin{array}{c}
2 m-2 k \\
m-k
\end{array}\right)\left(\left(\begin{array}{c}
n-1+m-k \\
2 m-2 k
\end{array}\right)+\left(\begin{array}{c}
n+1+m-k \\
2 m-2 k
\end{array}\right)\right) \\
= & 2 \sum_{k=0}^{m}\left(\begin{array}{c}
n+k \\
2 k
\end{array}\right) C_{k}\left(\begin{array}{c}
n+m-k \\
2 m-2 k
\end{array}\right)\left(\begin{array}{c}
2 m-2 k \\
m-k
\end{array}\right) \frac{(m+n-k)^{2}-n(2 m-2 k-1)}{(m+n-k)(n-m+k+1)} .
\end{aligned}
$$

By the Zeilberger algorithm we find that $u_{m}=c_{m}(n) / 2$ satisfies the recursion

$$
\begin{aligned}
& (m+2)(m+3)^{2}\left(m^{2}+5 m+6+4 n(n+1)\right) u_{m+2}+2 P(m, n) u_{m+1} \\
= & (m+2)\left((2 n+1)^{2}-m^{2}\right)\left(m^{2}+7 m+12+4 n(n+1)\right) u_{m}
\end{aligned}
$$

where $P(m, n)$ denotes the polynomial

$$
\begin{aligned}
& m^{5}+11 m^{4}+45 m^{3}+83 m^{2}+64 m+12+20 n^{4}-40 n^{3}-58 n^{2}-38 n \\
& -25 m n+m^{2} n+2 m^{3} n-33 m n^{2}+m^{2} n^{2}+2 m^{3} n^{2}-16 m n^{3}-8 m n^{4} .
\end{aligned}
$$


Clearly the coefficient of $x^{m}$ on the left-hand side of (3.6) coincides with

$$
d_{m}(n)=\sum_{k=0}^{m}\left(\begin{array}{c}
n+k \\
2 k
\end{array}\right)\left(\begin{array}{c}
2 k \\
k
\end{array}\right)^{2}\left(\begin{array}{c}
k+1 \\
m-k
\end{array}\right) \frac{2 k+1}{(k+1)^{2}}
$$

By the Zeilberger algorithm $u_{m}=d_{m}(n)$ also satisfies the recursion (3.7). Thus we have $d_{m}(n)=c_{m}(n)$ by induction on $m$. So (3.6) holds.

Proof of Theorem 1.2(ii). Write $p=2 n+1$. By (2.1),

$$
\sum_{k=0}^{n}\left(\begin{array}{c}
n+k \\
2 k
\end{array}\right)\left(\begin{array}{c}
2 k \\
k
\end{array}\right)\left(\begin{array}{c}
2 k \\
k+1
\end{array}\right) 2^{k}=\frac{n(n+1)}{2} S_{n}^{2}
$$

Thus, by (1.2) and (1.9) we have

$$
\begin{aligned}
\sum_{k=0}^{p-1} \frac{\left(\begin{array}{c}
2 k \\
k
\end{array}\right)^{2}\left(\begin{array}{c}
2 k \\
k+1
\end{array}\right)}{(-8)^{k}} & \equiv \sum_{k=0}^{n}\left(\begin{array}{c}
n+k \\
2 k
\end{array}\right)\left(\begin{array}{c}
2 k \\
k
\end{array}\right)\left(\begin{array}{c}
2 k \\
k+1
\end{array}\right) 2^{k} \\
& \equiv \frac{p^{2}-1}{8} 4\left(4 x^{2}-4 p\right)\left(\bmod p^{2}\right)
\end{aligned}
$$

and hence (1.12) holds.

Now we consider (1.13). Observe that

$$
\left(\begin{array}{c}
2 k \\
k+1
\end{array}\right)^{2}=\left(1-\frac{2 k+1}{(k+1)^{2}}\right)\left(\begin{array}{c}
2 k \\
k
\end{array}\right)^{2} \quad \text { for } k=0,1,2, \ldots
$$

and

$$
\left(\begin{array}{c}
2(p-1) \\
p-1
\end{array}\right)\left(\begin{array}{c}
2(p-1) \\
(p-1)+1
\end{array}\right)^{2}=\frac{p}{2 p-1}\left(\begin{array}{c}
2 p-1 \\
p-1
\end{array}\right)\left(\begin{array}{c}
2 p-2 \\
p-2
\end{array}\right)^{2} \equiv-p \quad\left(\bmod p^{2}\right)
$$

Thus we have

$$
\sum_{k=0}^{p-1} \frac{\left(\begin{array}{c}
2 k \\
k
\end{array}\right)\left(\begin{array}{c}
2 k \\
k+1
\end{array}\right)^{2}}{(-8)^{k}} \equiv-p+\sum_{k=0}^{n} \frac{\left(\begin{array}{c}
2 k \\
k
\end{array}\right)^{3}}{(-8)^{k}}-\sum_{k=0}^{n} \frac{(2 k+1)\left(\begin{array}{c}
2 k \\
k
\end{array}\right)^{3}}{(k+1)^{2}(-8)^{k}} \quad\left(\bmod p^{2}\right) .
$$

By (1.2) and (3.6) with $x=1$,

$$
\begin{aligned}
\sum_{k=0}^{n} \frac{(2 k+1)\left(\begin{array}{c}
2 k \\
k
\end{array}\right)^{3}}{(k+1)^{2}(-8)^{k}} & \equiv \sum_{k=0}^{n}\left(\begin{array}{c}
n+k \\
2 k
\end{array}\right)\left(\begin{array}{c}
2 k \\
k
\end{array}\right)^{2} \frac{(2 k+1) 2^{k}}{(k+1)^{2}} \\
& =\frac{S_{n}}{4}\left(D_{n-1}+D_{n+1}\right)\left(\bmod p^{2}\right)
\end{aligned}
$$


It is known (cf. [Sl] and [St, p. 191]) that

$$
(n+1) D_{n+1}=3(2 n+1) D_{n}-n D_{n-1} \quad \text { and } \quad D_{n+1}-3 D_{n}=2 n S_{n} .
$$

Thus

$$
\begin{aligned}
n\left(D_{n-1}+D_{n+1}\right) & =3(2 n+1) D_{n}-D_{n+1} \\
& =3(2 n+1) D_{n}-\left(3 D_{n}+2 n S_{n}\right)=2 n\left(3 D_{n}-S_{n}\right)
\end{aligned}
$$

and hence

$$
\sum_{k=0}^{n} \frac{(2 k+1)\left(\begin{array}{c}
2 k \\
k
\end{array}\right)^{3}}{(k+1)^{2}(-8)^{k}} \equiv \frac{S_{n}}{2}\left(3 D_{n}-S_{n}\right) \quad\left(\bmod p^{2}\right)
$$

With the help of (1.9) and (3.4), we have

$$
\frac{S_{n}}{2}\left(3 D_{n}-S_{n}\right) \equiv\left(2 x-\frac{p}{x}\right)\left(3\left(2 x-\frac{p}{2 x}\right)-\left(4 x-\frac{2 p}{x}\right)\right) \quad\left(\bmod p^{2}\right)
$$

and hence

$$
\sum_{k=0}^{n} \frac{(2 k+1)\left(\begin{array}{c}
2 k \\
k
\end{array}\right)^{3}}{(k+1)^{2}(-8)^{k}} \equiv 4 x^{2}-p \quad\left(\bmod p^{2}\right) .
$$

Combining this with (3.5) and (3.8), we immediately obtain (1.13).

\section{Proof of Theorem 1.3}

Lemma 4.1. Let $p$ be an odd prime. Then, for any $p$-adic integer $x \not \equiv$ $0,-1(\bmod p)$ we have

$$
\sum_{k=0}^{p-1}\left(\begin{array}{c}
2 k \\
k
\end{array}\right)^{3}\left(\frac{-x}{64}\right)^{k} \equiv\left(\frac{x+1}{p}\right) \sum_{k=0}^{p-1}\left(\begin{array}{c}
2 k \\
k
\end{array}\right)^{2}\left(\begin{array}{c}
4 k \\
2 k
\end{array}\right)\left(\frac{x}{64(x+1)^{2}}\right)^{k}(\bmod p) .
$$

Proof. Taking $n=(p-1) / 2$ in the following identity of MacMahon (see, e.g., $[\mathrm{G},(6.7)])$

$$
\sum_{k=0}^{n}\left(\begin{array}{l}
n \\
k
\end{array}\right)^{3} x^{k}=\sum_{k=0}^{n}\left(\begin{array}{c}
n+k \\
2 k
\end{array}\right)\left(\begin{array}{c}
2 k \\
k
\end{array}\right)\left(\begin{array}{c}
n-k \\
k
\end{array}\right) x^{k}(1+x)^{n-2 k}
$$

and noting (1.2) and the basic facts

$$
\left(\begin{array}{l}
n \\
k
\end{array}\right) \equiv\left(\begin{array}{c}
-1 / 2 \\
k
\end{array}\right)=\frac{\left(\begin{array}{c}
2 k \\
k
\end{array}\right)}{(-4)^{k}} \quad(\bmod p)
$$


and

$$
\left(\begin{array}{c}
n-k \\
k
\end{array}\right) \equiv\left(\begin{array}{c}
-1 / 2-k \\
k
\end{array}\right)=\frac{\left(\begin{array}{l}
4 k \\
2 k
\end{array}\right)}{(-4)^{k}} \quad(\bmod p),
$$

we immediately get (4.1).

Proof of Theorem 1.3. (i) For $d=0,1,2, \ldots$, we define

$$
f(d)=\sum_{k=0}^{p-1} \frac{\left(\begin{array}{c}
2 k \\
k+d
\end{array}\right)\left(\begin{array}{c}
2 k \\
k
\end{array}\right)\left(\begin{array}{c}
3 k \\
k
\end{array}\right)}{108^{k}}, \quad g(d)=\sum_{k=0}^{p-1} \frac{\left(\begin{array}{c}
2 k \\
k+d
\end{array}\right)\left(\begin{array}{c}
2 k \\
k
\end{array}\right)\left(\begin{array}{c}
4 k \\
2 k
\end{array}\right)}{256^{k}}
$$

and

$$
h(d)=\sum_{k=0}^{p-1} \frac{\left(\begin{array}{c}
2 k \\
k+d
\end{array}\right)\left(\begin{array}{c}
3 k \\
k
\end{array}\right)\left(\begin{array}{c}
6 k \\
3 k
\end{array}\right)}{12^{3 k}} .
$$

By the Zeilberger algorithm, we find the recursive relations:

$$
\begin{aligned}
& (3 d+1)(3 d+2) f(d)-(3 d+4)(3 d+5) f(d+2) \\
= & \frac{(3 p-1)(3 p-2)(d+1)}{108^{p-1} p}\left(\begin{array}{c}
2 p \\
p+d+1
\end{array}\right)\left(\begin{array}{c}
2 p-2 \\
p-1
\end{array}\right)\left(\begin{array}{c}
3 p-3 \\
p-1
\end{array}\right), \\
& (4 d+1)(4 d+3) g(d)-(4 d+5)(4 d+7) g(d+2) \\
= & \frac{(4 p-1)(4 p-3)(d+1)}{256^{p-1} p}\left(\begin{array}{c}
2 p \\
p+d+1
\end{array}\right)\left(\begin{array}{c}
2 p-2 \\
p-1
\end{array}\right)\left(\begin{array}{c}
4 p-4 \\
2 p-2
\end{array}\right),
\end{aligned}
$$

and

$$
\begin{aligned}
& (6 d+1)(6 d+5) h(d)-(6 d+7)(6 d+11) h(d+2) \\
= & \frac{(6 p-1)(6 p-5)(d+1)}{1728^{p-1} p}\left(\begin{array}{c}
2 p \\
p+d+1
\end{array}\right)\left(\begin{array}{c}
3 p-3 \\
p-1
\end{array}\right)\left(\begin{array}{c}
6 p-6 \\
3 p-3
\end{array}\right) .
\end{aligned}
$$

Recall that $\left(\begin{array}{c}2 p-2 \\ p-1\end{array}\right)=p C_{p-1} \equiv 0(\bmod p)$. Also,

$$
\begin{aligned}
& (3 p-2)\left(\begin{array}{c}
3 p-3 \\
p-1
\end{array}\right)=p\left(\begin{array}{c}
3 p-2 \\
p
\end{array}\right) \equiv 0(\bmod p), \\
& (4 p-3)\left(\begin{array}{c}
4 p-4 \\
2 p-2
\end{array}\right)=p\left(\begin{array}{c}
4 p-2 \\
2 p
\end{array}\right) \equiv 0(\bmod p), \\
& (6 p-5)\left(\begin{array}{c}
6 p-6 \\
3 p-3
\end{array}\right)=\frac{3 p(3 p-1)(3 p-2)}{(6 p-3)(6 p-4)}\left(\begin{array}{c}
6 p-3 \\
3 p
\end{array}\right) \equiv 0(\bmod p) .
\end{aligned}
$$

If $0 \leqslant d<p-1$, then

$$
\left(\begin{array}{c}
2 p \\
p+d+1
\end{array}\right)=\left(\begin{array}{c}
2 p \\
p-1-d
\end{array}\right) \equiv 0 \quad(\bmod p) .
$$


So, by (4.2)-(4.4), for any $d \in\{0, \ldots, p-1\}$ we have

$$
\begin{aligned}
& (3 d+1)(3 d+2) f(d) \equiv(3 d+4)(3 d+5) f(d+2)\left(\bmod p^{2}\right) \\
& (4 d+1)(4 d+3) g(d) \equiv(4 d+5)(4 d+7) g(d+2)\left(\bmod p^{2}\right) \\
& (6 d+1)(6 d+5) h(d) \equiv(6 d+7)(6 d+11) h(d+2)\left(\bmod p^{2}\right) .
\end{aligned}
$$

Fix $0 \leqslant d \leqslant p-1$. If $d \equiv\left(1+\left(\frac{p}{3}\right)\right) / 2(\bmod 2)$, then it is easy to verify that $\{3 d+1,3 d+2\} \cap\{p, 2 p\}=\emptyset$, hence $(3 d+1)(3 d+2) \not \equiv 0(\bmod p)$ and thus by (4.5) we have

$$
f(d+2) \equiv 0\left(\bmod p^{2}\right) \Longrightarrow f(d) \equiv 0\left(\bmod p^{2}\right) .
$$

If $d \equiv\left(1+\left(\frac{-2}{p}\right)\right) / 2(\bmod 2)$, then $\{4 d+1,4 d+3\} \cap\{p, 3 p\}=\emptyset$, hence $(4 d+1)(4 d+3) \not \equiv 0(\bmod p)$ and thus by $(4.6)$ we have

$$
g(d+2) \equiv 0\left(\bmod p^{2}\right) \Longrightarrow g(d) \equiv 0\left(\bmod p^{2}\right)
$$

If $d \equiv\left(1+\left(\frac{-1}{p}\right)\right) / 2(\bmod 2)$, then $\{6 d+1,6 d+3\} \cap\{p, 3 p, 5 p\}=\emptyset$, hence $(6 d+1)(6 d+3) \not \equiv 0(\bmod p)$ and thus $(4.7)$ yields

$$
h(d+2) \equiv 0\left(\bmod p^{2}\right) \Longrightarrow h(d) \equiv 0\left(\bmod p^{2}\right) .
$$

Since

$$
f(p)=f(p+1)=g(p)=g(p+1)=h(p)=h(p+1)=0,
$$

by the last paragraph, for every $d=p+1, p, \ldots, 0$ we have the desired (1.19)-(1.21).

(ii) Assume that $p \equiv 3(\bmod 8)$ and $p=x^{2}+2 y^{2}$ with $x, y \in \mathbb{Z}$. Since $4 x^{2} \not \equiv 0(\bmod p)$ and Mortenson $[\mathrm{M}]$ already proved that the squares of both sides of $(1.22)$ are congruent modulo $p^{2},(1.22)$ is reduced to its $\bmod$ $p$ form. Applying (4.1) with $x=1$ we get

$$
\sum_{k=0}^{p-1} \frac{\left(\begin{array}{c}
2 k \\
k
\end{array}\right)^{3}}{(-64)^{k}} \equiv\left(\frac{2}{p}\right) \sum_{k=0}^{p-1} \frac{\left(\begin{array}{c}
2 k \\
k
\end{array}\right)^{2}\left(\begin{array}{c}
4 k \\
2 k
\end{array}\right)}{256^{k}} \quad(\bmod p) .
$$

By $[\mathrm{A}$, Theorem 5(3)], we have

$$
\left(\frac{-1}{p}\right) \sum_{k=0}^{n}\left(\begin{array}{l}
n \\
k
\end{array}\right)^{2}\left(\begin{array}{c}
n+k \\
k
\end{array}\right)(-1)^{k} \equiv 4 x^{2}-2 p \quad(\bmod p),
$$


where $n=(p-1) / 2$. For $k=0, \ldots, n$ clearly

$$
\begin{aligned}
\left(\begin{array}{l}
n \\
k
\end{array}\right)^{2}\left(\begin{array}{c}
n+k \\
k
\end{array}\right)(-1)^{k} & =\left(\begin{array}{c}
(p-1) / 2 \\
k
\end{array}\right)^{2}\left(\begin{array}{c}
-(p+1) / 2 \\
k
\end{array}\right) \\
& \equiv\left(\begin{array}{c}
-1 / 2 \\
k
\end{array}\right)^{3}=\frac{\left(\begin{array}{c}
2 k \\
k
\end{array}\right)^{3}}{(-64)^{k}}(\bmod p),
\end{aligned}
$$

therefore

$$
\sum_{k=0}^{p-1} \frac{\left(\begin{array}{c}
2 k \\
k
\end{array}\right)^{3}}{(-64)^{k}} \equiv\left(\frac{-1}{p}\right)\left(4 x^{2}-2 p\right) \quad(\bmod p)
$$

and hence (1.22) follows.

(iii) Finally we suppose $p \equiv 5(\bmod 12)$ and write $p=x^{2}+y^{2}$ with $x$ odd and $y$ even. Once again it suffices to show the $\bmod p$ form of (1.23) in view of Mortenson's work [M]. As Z.-H. Sun observed,

$$
\left(\begin{array}{c}
(p-5) / 6+k \\
2 k
\end{array}\right)\left(\begin{array}{c}
2 k \\
k
\end{array}\right) \equiv\left(\begin{array}{c}
k-5 / 6 \\
2 k
\end{array}\right)\left(\begin{array}{c}
2 k \\
k
\end{array}\right)=\frac{\left(\begin{array}{c}
3 k \\
k
\end{array}\right)\left(\begin{array}{c}
6 k \\
3 k
\end{array}\right)}{(-432)^{k}} \quad(\bmod p)
$$

for all $k=0,1,2, \ldots$ If $p / 6<k<p / 3$ then $p \mid\left(\begin{array}{c}6 k \\ 3 k\end{array}\right)$; if $p / 3<k<p / 2$ then $p \mid\left(\begin{array}{c}3 k \\ k\end{array}\right)$; if $p / 2<k<p$ then $p \mid\left(\begin{array}{c}2 k \\ k\end{array}\right)$. Thus

$$
\begin{aligned}
\sum_{k=0}^{p-1} \frac{\left(\begin{array}{c}
2 k \\
k
\end{array}\right)\left(\begin{array}{c}
3 k \\
k
\end{array}\right)\left(\begin{array}{c}
6 k \\
3 k
\end{array}\right)}{12^{3 k}} & \equiv \sum_{k=0}^{(p-5) / 6}\left(\begin{array}{c}
(p-5) / 6+k \\
2 k
\end{array}\right)\left(\begin{array}{c}
2 k \\
k
\end{array}\right)^{2}\left(-\frac{1}{4}\right)^{k} \\
& =D_{2 n}\left(-\frac{1}{2}\right)^{2}(\bmod p) \quad(\text { by }(1.3)),
\end{aligned}
$$

where $n=(p-5) / 12$. Note that

$$
D_{2 n}\left(-\frac{1}{2}\right)=\frac{1}{(-4)^{n}}\left(\begin{array}{c}
2 n \\
n
\end{array}\right)
$$

by $[\mathrm{G},(3.133)$ and $(3.135)]$, and

$$
\left(\begin{array}{c}
(p-1) / 2 \\
(p-1) / 4
\end{array}\right) \equiv 12(-432)^{n}\left(\begin{array}{c}
2 n \\
n
\end{array}\right) \quad(\bmod p)
$$

by P. Morton [Mo]. Therefore

$D_{2 n}\left(-\frac{1}{2}\right)^{2}=\frac{1}{16^{n}}\left(\begin{array}{c}2 n \\ n\end{array}\right)^{2} \equiv \frac{\left(\begin{array}{c}(p-1) / 2 \\ (p-1) / 4\end{array}\right)^{2}}{12^{6 n+2}} \equiv\left(\frac{12}{p}\right)\left(\begin{array}{l}(p-1) / 2 \\ (p-1) / 4\end{array}\right)^{2} \quad(\bmod p)$.

Thus, by applying Gauss' congruence $\left(\begin{array}{c}(p-1) / 2 \\ (p-1) / 4\end{array}\right) \equiv 2 x(\bmod p)(\mathrm{cf} .[\mathrm{BEW}$, (9.0.1)] or $[\mathrm{HW}])$ we immediately get the $\bmod p$ form of (1.23) from the above.

The proof of Theorem 1.3 is now complete.

Acknowledgment. The author would like to thank the referee for helpful comments. 


\section{REFERENCES}

[A] S. Ahlgren, Gaussian hypergeometric series and combinatorial congruences, in: Symbolic Computation, Number Theory, Special Functions, Physics and Combinatorics (Gainesville, FI, 1999), pp. 1-12, Dev. Math., Vol. 4, Kluwer, Dordrecht, 2001.

[BEW] B. C. Berndt, R. J. Evans and K. S. Williams, Gauss and Jacobi Sums, John Wiley \& Sons, 1998.

[CHV] J. S. Caughman, C. R. Haithcock and J. J. P. Veerman, A note on lattice chains and Delannoy numbers, Discrete Math. 308 (2008), 2623-2628.

[CDE] S. Chowla, B. Dwork and R. J. Evans, On the mod $p^{2}$ determination of $\left(\begin{array}{l}(p-1) / 2 \\ (p-1) / 4\end{array}\right)$, J. Number Theory 24 (1986), 188-196.

[C] D. A. Cox, Primes of the Form $x^{2}+n y^{2}$, John Wiley \& Sons, 1989.

[G] H. W. Gould, Combinatorial Identities, Morgantown Printing and Binding Co., 1972 .

[GZ] J. Guillera and W. Zudilin, "Divergent" Ramanujan-type supercongruences, Proc. Amer. Math. Soc. 140 (2012), 765-777.

[HW] R. H. Hudson and K. S. Williams, Binomial coefficients and Jacobi sums, Trans. Amer. Math. Soc. 281 (1984), 431-505.

[I] T. Ishikawa, Super congruence for the Apéry numbers, Nagoya Math. J. 118 (1990), 195-202.

[KF] F. Klein and R. Fricke, Vorlesungen uber die Theorie der elliptischen Modulfunktionen, Teubner, Leipzig, 1892.

[M] E. Mortenson, Supercongruences for truncated ${ }_{n+1} F_{n}$ hypergeometric series with applications to certain weight three newforms, Proc. Amer. Math. Soc. 133 (2005), 321-330.

[Mo] P. Morton, Explicit identities for invariants of elliptic curves, J. Number Theory 120 (2006), 234-271.

[O] K. Ono, Web of Modularity: Arithmetic of the Coefficients of Modular Forms and q-series, Amer. Math. Soc., Providence, R.I., 2003.

[PWZ] M. Petkovšek, H. S. Wilf and D. Zeilberger, $A=B$, A K Peters, Wellesley, 1996.

[RV] F. Rodriguez-Villegas, Hypergeometric families of Calabi-Yau manifolds, in: Calabi-Yau Varieties and Mirror Symmetry (Toronto, ON, 2001), pp. 223-231, Fields Inst. Commun., 38, Amer. Math. Soc., Providence, RI, 2003.

[Sl] N. J. A. Sloane, Sequences A001850 and A006318 in OEIS (On-Line Encyclopedia of Integer Sequences), http://oeis.org.

[St] R. P. Stanley, Enumerative Combinatorics, Vol. 2, Cambridge Univ. Press, Cambridge, 1999.

[SB] J. Stienstra and F. Beukers, On the Picard-Fuchs equation and the formal Brauer group of certain elliptic K3-surfaces, Math. Ann. 271 (1985), 269-304.

[S1] Z.-H. Sun, Congruences concerning Legendre polynomials, Proc. Amer. Math. Soc. 139 (2011), 1915-1929.

[S2] Z.-H. Sun, Congruences concerning Legendre polynomials (II), arXiv:1012.3898.

[Su1] Z.-W. Sun, Binomial coefficients, Catalan numbers and Lucas quotients, Sci. China Math. 53 (2010), 2473-2488.

[Su2] Z.-W. Sun, On congruences related to central binomial coefficients, J. Number Theory 131 (2011), 2219-2238.

[Su3] Z.-W. Sun, Super congruences and Euler numbers, Sci. China Math. 54 (2011), 2509-2535.

[Su4] Z.-W. Sun, On Delannoy numbers and Schroder numbers, J. Number Theory 131 (2011), 2387-2397. 
[vH] L. van Hamme, Some conjectures concerning partial sums of generalized hypergeometric series, in: p-adic Functional Analysis (Nijmegen, 1996), pp. 223-236, Lecture Notes in Pure and Appl. Math., Vol. 192, Dekker, 1997.

[Z] D. Zeilberger, A fast algorithm for proving terminating hypergeometric series identities, Discrete Math. 80 (1990), 207-211. 